\title{
ON THE ZEROS OF THE DERIVATIVES OF SOME ENTIRE FUNCTIONS OF FINITE ORDER
}

\author{
by ROBERT M. GETHNER
}

(Received 12th October 1984)

\section{Introduction}

Given a function $f$ meromorphic in the plane, and complex numbers $w$ and $a$, call $w$ an a-point of $f^{(k)}$ if $f^{(k)}(w)=a$. Denote by $\Lambda(a, f)$ the set of $z \in \mathbb{C}$ such that every neighborhood of $z$ contains $a$-points of infinitely many of the functions $f^{(k)}$. Adapting the terminology of Pólya [16], who introduced the sets $\Lambda(a, f)$ in [15], we call $\Lambda(a, f)$ the final set of $f$ with respect to $a$.

Pólya showed that if $f$ has at least one pole, then its final sets are remarkably regular. For each pole $A$ of $f$, define the shire of $A$ to be the set of all points closer to $A$ than to any other pole of $f$.

Pólya "shire" theorem. If $a \in \mathbb{C}$ and $f$ is a function meromorphic in $\mathbb{C}$ having at least one pole, then $\Lambda(a, f)$ is the union of the boundaries of the shires of all poles of $f$.

Pólya paraphrased his theorem in the case $a=0$ by stating that the poles of a meromorphic function repel the zeros of its higher derivatives.

The object of this paper is to establish a result analogous to the above theorem for a certain class of entire functions of finite order. This result (Theorem 1) enables us to determine, in Section 8, the final sets of a variety of functions, including some of fractional order (e.g. the Mittag-Leffler functions and a class of Lindelöf functions of genus zero) and some of maximal or minimal type, as well as certain sums of exponential powers.

Pólya, McLeod [13, 14], and Edrei $[4,5]$ have determined the final sets of various entire functions. A common feature of the functions examined by these authors is that the final set lies, roughly speaking, as far as possible from certain rays on which $f$ has its sharpest growth. These rays therefore seem to repel the zeros of the derivatives, just as the poles do in the theorem of Pólya quoted above. This similarity suggests the existence of a "shire theorem" for entire functions, in which the set of poles is replaced by a set of rays. Theorem 1 is such a theorem.

In Section 2 we develop the definitions, including that of shire, needed to state (in Section 3) Theorem 1 and some related results. The proof of Theorem 1 takes up Sections 4 through 7. After some preliminaries (Section 4), we use a saddle point argument adapted from the work of McLeod and Edrei to derive bounds on the derivatives of the functions $f$ under consideration (Sections 5 and 6). An examination of these bounds shows that $\Lambda(a, f)$ is contained in the union of the boundaries of the shires. In Section 7 we determine asymptotically the number of $a$-points of $f^{(k)}$ in small 
discs centred at points on the boundaries, using a slight modification of an argument in [5]. This determination shows that every boundary point is contained in $\Lambda(a, f)$. Also in Section 7, we prove the other theorems stated in Section 3.

A description of saddle point methods may be found in [3].

This paper is based on a special case of my doctoral dissertation. I am grateful to my advisor, Professor Simon Hellerstein, whose skillful guidance made this work possible. I would also like to thank Professor Linda Sons for her helpful comments on a draft of this paper.

\section{Notation and definitions}

The following notations and definitions are used throughout the paper. We take $x, r$, $t$, and $\theta$ to be real variables. The letters $N$ and $C$ denote positive constants, possibly different at each occurrence. By $A \sim B$ (" $A$ is asymptotic to $B$ ") we mean $A / B \rightarrow 1$. All real powers of complex numbers denote the principal branch. For $z \in \mathbb{C}$ and $\rho>0$, we set

$$
D(z ; \rho)=\{w \in \mathbb{C}:|w-z|<\rho\} .
$$

We employ the standard notations of Nevanlinna Theory, such as $M(r, f), m(r, f)$, and $n(r, a, f)$.

Given $N_{0}>0$ and $\sigma, 0<\sigma<\pi$, we will say that a function $L$ holomorphic in $\left\{|\arg z|<\sigma,|z|>N_{0}\right\}$ is slowly varying if $L(x)>0$ for $x>N_{0}$, and

$$
z \frac{L^{\prime}(z)}{L(z)} \rightarrow 0 \quad(|z| \rightarrow \infty)
$$

uniformly for $|\arg z|<\sigma$.

\section{Remarks}

(a) It is a consequence of Lemma 1(c) (Section 4) that the function $x \rightarrow L(x)$ is "slowly increasing" ([11, p. 32]).

(b) Put $\log _{1}=\log$, and $\log _{p}=\log _{\log } \log ^{1}(p=2,3,4, \ldots)$. By induction, $\log _{p}$ is slowly varying for each $p$. Moreover, a product of slowly varying functions is again slowly varying, as is a real power of such a function. Hence if $A(1), \ldots, A(P) \in \mathbb{Z}^{+}$and $B(1), \ldots, B(P) \in \mathbb{R}$, then the function

$$
z \rightarrow\left(\log _{A(1)} z\right)^{B(1)} \ldots\left(\log _{A(P)} z\right)^{B(P)}
$$

is slowly varying. Some examples not of the form (2.2) are

$$
z \rightarrow\left\{\begin{array}{l}
\exp \left(\log _{2} z\right)^{2} \\
\exp \exp \left(\log _{3} z\right)^{3} . \\
1+z^{-1}
\end{array}\right.
$$


We now make precise the notions of "sharp growth on rays" and "shire" referred to in the introduction. Fix a positive integer $J$, complex numbers $v_{1}, \ldots, v_{J}$, and distinct real numbers $\theta_{1}, \ldots, \theta_{J}$ satisfying

$$
-\pi<\theta_{j} \leqq \pi \quad(j=1, \ldots, J)
$$

For $j=1, \ldots, J$, put

$$
\begin{gathered}
\beta_{j}(z)=e^{-i \theta_{j}}\left(z-v_{j}\right) \quad(z \in \mathbb{C}), \\
S_{j}(\sigma)=\beta_{j}^{-1}(\{|\arg z|<\sigma\}) \quad(0<\sigma<\pi), \\
R_{j}=R\left(v_{j}, \theta_{j}\right)=\beta_{j}^{-1}\left(\mathbb{R}^{+}\right),
\end{gathered}
$$

and

$$
\Omega_{j}=\left\{z \in \mathbb{C}: \operatorname{Re} \beta_{j}(z)>\operatorname{Re} \beta_{q}(z) \text { for all } q \neq j\right\}
$$

Call the set $\Omega_{j}$ the shire of the ray $R_{j}$.

Suppose $\lambda>0, N_{0}>0$, and $0<\eta<\min \{(\pi / \lambda), \pi\}$. Suppose $L$ is slowly varying on $\left\{|\arg z|<\eta,|z|>N_{0}\right\}$. We will say that an entire function $f$ has maximal rays $\left\{R_{j}\right\}_{j=1}^{J}$ (relative to $L$ and $\lambda$ ) if both the following conditions hold.

(a) For $j=1, \ldots, J$,

$$
\log \left|f(z) \exp \left\{-\left[\beta_{j}(z)\right]^{\lambda} L\left[\beta_{j}(z)\right]\right\}\right|=o(1) \cdot|z|^{\lambda-1} L(|z|) \quad(|z| \rightarrow \infty),
$$

uniformly for $z \in S_{j}(\eta)$.

(b) There exist $N>0$ and $\kappa, 0<\kappa<1$, such that if $|z|>N$ and $z \in \mathbb{C}-\bigcup_{j=1}^{J} S_{j}(\eta)$, then

$$
\log |f(z)|<\kappa|z|^{\lambda} L(|z|)
$$

\section{Remarks}

(a) Condition (a) above implies that $f$ has at most finitely many zeros in $\bigcup_{j=1}^{J} S_{j}(\eta)$.

(b) It follows from (2.8). (2.4), and (4.3) that

$$
\frac{\log \left|f \circ \beta_{j}^{-1}\left(r e^{i \theta}\right)\right|}{r^{\lambda} L(r)} \rightarrow \cos \theta \lambda \quad(r \rightarrow \infty),
$$

uniformly for $|\theta|<\eta$. Furthermore, by Lemma 1(a) and (2.1), the function

$$
\lambda(r)=\frac{\log \left[r^{\lambda} L(r)\right]}{\log r}
$$

is a "proximate order" ([11, p. 32]). Thus $f \circ \beta_{j}^{-1}$ is of "completely regular growth", order $\lambda$, in the angle $(-\eta, \eta)([11$, p. 139]). In addition, in view of (2.9), $f$ 
is of order $\lambda$; in fact,

$$
\frac{\log \log M(r, f)}{\log r} \rightarrow \lambda \quad(r \rightarrow \infty)
$$

(c) The class of functions having maximal rays contains the class $\mathscr{F}$ considered by McLeod [14].

\section{Statement of main results}

Theorems 1 through 3 to follow give our main results. We begin with the "shire theorem".

Theorem 1. If $f$ is an entire function having maximal rays $\left\{R_{j}\right\}_{j=1}^{J}$ relative to some $\lambda>1$, and the sets $\left\{\Omega_{j}\right\}_{j=1}^{J}$ are given by (2.7), then, for each $a \in \mathbb{C}$,

$$
\Lambda(a, f)=\bigcup_{j=1}^{J} \partial \Omega_{j}
$$

\section{Remarks on Theorem 1.}

(a) In particular, if $J=1$, then $\Lambda(a, f)$ is void.

(b) We shall see (Corollary; Section 6) that the a-points of $f^{(k)}$ (large $k$ ) avoid the shires in the following strong sense: For each compact $K \subset \bigcup_{j=1}^{J} \Omega_{j},\left|f^{(k)}(u)\right| \rightarrow \infty$ $(k \rightarrow \infty)$, uniformly for $u \in K$.

The next theorem gives asymptotically, for a certain class of functions having maximal rays, the number of $a$-points near points $z$ on the boundaries of the shires. We may assume without loss of generality that $z=0$. We may also assume, after a permutation of indices, first, that there exists $J^{\prime}, 1 \leqq J^{\prime} \leqq J$, such that $0 \in \partial \Omega_{j}$ if and only if $j \leqq J^{\prime}$, and second, that $\theta_{1}<\theta_{2}<\cdots<\theta_{J^{\prime}}$

Theorem 2. Suppose $\lambda>1, \mu>\frac{1}{2}$, and $N_{0}>0$. Let $L$ be a slowly varying function, defined for all $x>N_{0}$, such that

$$
(\log x)^{\mu} x \frac{L^{\prime}(x)}{L(x)}=O(1) \quad(x \rightarrow \infty) .
$$

Suppose $f$ has maximal rays $\left\{R\left(v_{j}, \theta_{j}\right)\right\}_{j=1}^{J}$ relative to $L$ and $\lambda$.

Suppose further that there is a $J^{\prime}, 1 \leqq J^{\prime} \leqq J$, such that $0 \in \partial \Omega_{j}$ if and only if $j \leqq J^{\prime}$, and assume that $\theta_{1}<\theta_{2}<\cdots<\theta_{J^{\prime}}$. Put $\theta_{0}=\theta_{J^{\prime}}-2 \pi$. For $k>\lambda N_{0}^{\lambda}$, put

$$
t_{k}=\lambda^{1 / \lambda} k^{1-1 / \lambda}\left\{L\left[\left(\frac{k}{\lambda}\right)^{1 / \lambda}\right]\right\}^{1 / \lambda} .
$$


Then there exists $\rho_{0}>0$ such that, for each $\rho, 0<\rho<\rho_{0}$, and each $a \in \mathbb{C}$,

$$
n\left(\rho, a, f^{(k)}\right) \sim\left[\frac{1}{\pi} \sum_{j=1}^{J \prime} \sin \left(\frac{\theta_{j}-\theta_{j-1}}{2}\right)\right] t_{k} \rho \quad(k \rightarrow \infty) .
$$

Remark. It may be that the conclusion of Theorem 2 holds even if $L$ does not satisfy (3.1), but I am unable to prove this. The proof of Theorem 1 does however, yield a less explicit asymptotic formula (7.23) valid for all slowly varying $L$. It is easy to check that the functions (2.2) satisfy (3.1).

The last theorem of this section gives some information about functions of order no greater than one, having exactly one maximal ray.

Theorem 3. Suppose $f$ has maximal rays $\left\{R_{j}\right\}_{j=1}^{J}$, relative to $\lambda$, with $J=1$. If $\lambda=1$, then $\Lambda(0, f)$ is void. If $\lambda<1$, then, for each $a \in \mathbb{C}, \Lambda(a, f)$ is void.

Remark. If $f(z)=e^{z}$, then $\Lambda(a, f)$ is void if and only if $a=0$.

\section{Preliminary Lemmas}

We develop here the basic tools for the proofs of our main results. In our first lemma we collect a number of facts about slowly varying functions.

Lemma 1. Suppose $0<\sigma<\pi$ and $N_{0}>0$. Let $L$ be slowly varying on $\left\{|\arg z|<\sigma,|z|>N_{0}\right\}$. Fix $\lambda>0$ and $\gamma$ real. Then:

(a) For each $\varepsilon>0$ there is an $N>0$ such that

$$
x^{-\varepsilon}<L(x)<x^{\varepsilon} \quad(x>N) .
$$

(b) There exists $N>0$ such that $x^{\lambda} L(x)$ and $x(d / d x)\left[x^{\lambda} L(x)\right]$ increase with $x$ for $x>N$. Furthermore, both quantities tend to infinity with $x$.

(c) For each $\mathrm{C}>0$,

$$
L(z+w) \sim L(z) \quad(|z|,|z+w| \rightarrow \infty),
$$

uniformly for $z$ such that $|\arg z|<\sigma$ and $w$ such that $|w|<C|z|$ and $|\arg (z+w)|<\sigma$.

(d) Fix $\varepsilon>0$. Suppose $H$ is holomorphic in the region $\left\{|\arg z|<\sigma,|z|>N_{0}\right\}$ and satisfies, for all $z$ in that region,

$$
|H(z)|<\varepsilon\left|z^{\gamma} L(z)\right| .
$$

Then there exist $N, C_{1}, C_{2}>0$ such that

$$
\left|\frac{H^{(q)}(z)}{q !}\right|<\varepsilon C_{1}\left|\left(C_{2} z\right)^{\gamma-q} L(z)\right|
$$

for all integers $q \geqq 0$ and all $z$ in the region $\left\{|\arg z|<\frac{3}{4} \sigma,|z|>N\right\}$. 
(e) For each $C>0$, both the following relations hold as $|z| \rightarrow \infty$, uniformly for all $z$ and $w$ such that $|\arg z|<\frac{3}{4} \sigma$ and $|w|<C$ :

$$
\begin{gathered}
(z+w)^{\gamma} L(z+w)=z^{\gamma} L(z)+\gamma z^{\gamma-1} L(z) w+o(1) \cdot z^{\gamma-1} L(z) \\
(z+w)^{\gamma} L^{\prime}(z+w)=z^{\gamma} L^{\prime}(z)+o(1) \cdot z^{\gamma-2} L(z) .
\end{gathered}
$$

(f) There exist $N_{1}>N_{0}$ and $\sigma_{1}, 0<\sigma_{1}<\sigma$, such that if $r>N_{1}$ and $-\sigma_{1}<\omega<\sigma_{1}$, then

$$
\operatorname{re}\left\{r^{\lambda} e^{i \omega \lambda} L\left(r e^{i \omega}\right)\right\} \leqq r^{\lambda} L(r) .
$$

Proof. Part (a) follows quickly from (2.1) and the equation

$$
\log L(x)=\log L(N)+\int_{N}^{x} \frac{L^{\prime}(t)}{L(t)} d t .
$$

To prove (c), we choose $r>0$ and $\theta, 0 \leqq \theta<\sigma$, and let $\Gamma$ be the contour $\left\{r e^{i t}: 0 \leqq t \leqq \theta\right\}$. From (2.1) and the equation

$$
\log L\left(r e^{i \theta}\right)-\log L(r)=\int_{\Gamma} \frac{L^{\prime}(\xi)}{L(\xi)} d \xi
$$

we deduce that

$$
L\left(r e^{i \theta}\right) \sim L(r) \quad(r \rightarrow \infty),
$$

uniformly in $\theta,-\sigma<\theta<\sigma$. Hence we may assume that $z$ and $z+w$ are positive real numbers. Another application of the fundamental theorem of calculus gives (c) for such $z$ and $w$.

To prove (d), we choose a suitably small $\tau>0$ and apply Cauchy's Estimates to $H$ on the disc $D(z ; \tau|z|)$. Part (d) then follows from part (c).

We proceed to the proof of (e). Using (2.1), and (d) with $H=L^{\prime}$, we find that, for each $\varepsilon>0$, there exist $N, C_{1}, C_{2}>0$ such that

$$
\left|\frac{L^{(q+1)}(z)}{q !}\right| \leqq \varepsilon C_{1}\left|\left(C_{2} z\right)^{-q-1} L(z)\right|
$$

for all $z$ such that $|\arg z|<\frac{3}{4} \sigma$ and $|z|>N$, and all integers $q \geqq 0$. Integrating $L^{\prime}$ over the segment from $z$ to $z+w$ and using (4.4) with $q=0$, we have, for each fixed $\varepsilon>0$,

$$
|L(z+w)-L(z)| \leqq \int_{z}^{z+w} \varepsilon C_{1} C_{2}^{-1}\left|\frac{L(t)}{t}\right| d t .
$$

Thus, if $|w|<C$, we may apply (c) to obtain

$$
L(z+w)=L(z)+o(1) \cdot z^{-1} L(z) \quad(|z| \rightarrow \infty),
$$


uniformly for $|w|<C$ and $|\arg z| \leqq \frac{3}{4} \sigma$. To complete the proof of (4.1), we expand $(z+w)^{\gamma}$ using the binomial theorem and multiply the resulting equation by (4.5). A similar argument gives (4.2).

To prove (f), we write

$$
L\left(r e^{i \omega}\right)=L\left[r+r\left(e^{i \omega}-1\right)\right]=L(r)+r L^{\prime}(r)\left(e^{i \omega}-1\right)+\sum_{p=2}^{\infty} r^{p} \frac{L^{(p)}(r)}{p !}\left(e^{i \omega}-1\right)^{p}
$$

Using (4.4), (2.1), and the Taylor Series for $e^{i \omega}$, we obtain

$$
L\left(r e^{i \omega}\right)=L(r)+i \omega r L^{\prime}(r)+o(1) \cdot \omega^{2} L(r) \quad(r \rightarrow \infty, \omega \rightarrow 0) .
$$

Taking real and imaginary parts of the quantities $L\left(r e^{i \omega}\right)$ and $r^{\lambda} e^{i \omega \lambda}$, we conclude with the aid of (2.1) and the series for $\sin \lambda \omega$ and $\cos \lambda \omega$ that

$$
\operatorname{re}\left\{r^{\lambda} e^{i \omega \lambda} L\left(r e^{i \omega}\right)\right\}=r^{\lambda} L(r)\left[1-\left(\frac{\lambda^{2}}{2}+o(1)\right) \omega^{2}\right] \quad(r \rightarrow \infty, \omega \rightarrow 0),
$$

from which (f) follows immediately.

It remains to verify (b). From (2.1) and (4.4) (with $q=1$ ) we deduce that, as $x \rightarrow \infty$,

$$
x \frac{d}{d x}\left[x^{\lambda} L(x)\right] \sim \lambda x^{\lambda} L(x)
$$

and

$$
\frac{d}{d x}\left[x \frac{d}{d x}\left[x^{\lambda} L(x)\right]\right] \sim \lambda^{2} x^{\lambda-1} L(x)
$$

Part (b) follows from (4.6), (4.7), and part (a). This completes the proof of Lemma 1.

The next lemma aids our analysis of $f(z)$ for $z \in S_{j}(\eta)$.

Lemma 2. Suppose $\lambda, N_{0}>0$ and $0<\eta<\pi$. Let $\phi$ be a function holomorphic in the region $\left\{|\arg z|<\eta,|z|>N_{0}\right\}$, satisfying

$$
\frac{\log |\phi(z)|}{|z|^{\lambda-1} L(z \mid)} \rightarrow 0 \quad(|z| \rightarrow \infty ;|\arg z|<\eta)
$$

Then, as $|z| \rightarrow \infty$ with $|\arg z|<\frac{1}{2} \eta$,

$$
z \frac{\phi^{\prime}(z)}{\phi(z)}=o(1) \cdot|z|^{2-1} L(|z|)
$$

and

$$
z\left[z \frac{\phi^{\prime}(z)}{\phi(z)}\right]^{\prime}=o(1) \cdot|z|^{\lambda-1} L(z \mid)
$$


Proof. Choose $N$ and $\tau, 0<\tau<1$, such that if $|z|>N$ and $|\arg z|<\frac{3}{4} \eta$, then $\overline{D(z ; 2 \tau|z|)} \subset\{|\arg w|<\eta\}$. Fixing such a $z$, define $\Phi(w)$ (for $w \in \overline{D(z ; 2 \tau|z|))}$ to be the holomorphic branch of $\log (\phi(w) / \phi(z))$ satisfying $\Phi(z)=0$. By Cauchy's Estimates on $D(z ; \tau|z|)$ and the Borel-Carathéodory Inequality [18, p. 174],

$$
\tau\left|z \Phi^{\prime}(z)\right| \leqq 2 \max \left\{\log \left|\frac{\phi(w)}{\phi(z)}\right|:|w-z|=2 \tau|z|\right\} .
$$

Now, allowing $z$ to vary, we deduce from the above inequality, along with (4.8), (4.8) with $z$ replaced by $w$, and Lemma $1(\mathrm{c})$, that

$$
\left|z \Phi^{\prime}(z)\right|=o(1) \cdot|z|^{\lambda-1} \cdot L(|z|) \quad\left(|z| \rightarrow \infty ;|\arg z|<\frac{3}{4} \eta\right),
$$

from which (4.9) follows immediately. Relation (4.10) is a consequence of (4.10a) and Lemma 1 (d) with $H(z)=z \Phi^{\prime}(z)$ and $\sigma=\frac{3}{4} \eta$.

We will use the next theorem to estimate the derivatives of functions having maximal rays. With a change in notation, it is a specialization of a theorem of McLeod, which is in turn an extension of a result of Hayman [8]. The proof of McLeod's theorem is given in [14].

Theorem 4. Let $f$ be an entire function. For $u, z \in \mathbb{C}$, put

$$
\begin{aligned}
& f_{u}(z)=f(u+z), \\
& A_{u}(z)=z \frac{f_{u}^{\prime}(z)}{f_{u}(z)},
\end{aligned}
$$

and

$$
B_{u}(z)=z A_{u}^{\prime}(z)
$$

Let $C_{1}, C_{2}, r_{1}>0$ be constants, and let $K \subset \mathbb{C}$ be a compact set. Suppose both the following statements are true.

(a) For each $r>r_{1}$ and $u \in K$, there is a point $\zeta_{u}(r) \in \mathbb{C}$, with $\left|\zeta_{u}(r)\right|=r$, such that

$$
\begin{array}{cl}
\frac{\left[\operatorname{im} A_{u}\left(\zeta_{u}(r)\right)\right]^{2}}{\operatorname{re~} B_{u}\left(\zeta_{u}(r)\right)} \leqq C_{1} & \left(r>r_{1}, u \in K\right), \\
\left|\frac{B_{u}\left(\zeta_{u}(r)\right)}{\operatorname{re} B_{u}\left(\zeta_{u}(r)\right)}\right| \leqq C_{2} & \left(r>r_{1}, u \in K\right),
\end{array}
$$

and, uniformly for $u \in K$,

$$
\left|B_{u}\left(\zeta_{u}(r)\right)\right| \rightarrow \infty \quad(r \rightarrow \infty)
$$


(b) For each $u \in K$ there is a function $\Delta_{u^{\prime}}:\left(r_{1}, \infty\right) \rightarrow(0, \pi)$ such that, as $r \rightarrow \infty$,

$$
f_{u}\left(\zeta_{u}(r) e^{i t}\right) \sim f_{u}\left(\zeta_{u}(r)\right) \cdot \exp \left\{i t A_{u}\left(\zeta_{u}(r)\right)-\frac{1}{2} t^{2} B_{u}\left(\zeta_{u}(r)\right)\right\}
$$

uniformly for $u \in K$ and $|t| \leqq \Delta_{u}(r)$, whereas

$$
f_{u}\left(\zeta_{u}(r) e^{i t}\right)=o\left[\frac{f_{u}\left(\zeta_{u}(r)\right)}{\left(B_{u}\left(\zeta_{u}(r)\right)\right)^{1 / 2}}\right]
$$

uniformly for $u \in K$ and $\Delta_{u}(r) \leqq|t| \leqq \pi$. Then: As $r \rightarrow \infty$,

$$
\frac{f^{(k)}(u)}{k !}\left[\zeta_{u}(r)\right]^{k}=\frac{f_{u}\left(\zeta_{u}(r)\right)}{\left[2 \pi B_{u}\left(\zeta_{u}(r)\right)\right]^{1 / 2}}\left\{\exp \left[-\frac{\left(A_{u}\left(\zeta_{u}(r)\right)-k\right)^{2}}{2 B_{u}\left(\zeta_{u}(r)\right)}\right]+o(1)\right\}
$$

uniformly for $u \in K$ and $k \in \mathbb{Z}^{+}$.

\section{The Saddle Point Equation}

We assume throughout Sections 5, 6, and 7, that an entire function $f$ has been fixed, having maximal rays $\left\{R_{j}\right\}_{j=1}^{J}$ relative to some $\lambda>0$ and some slowly varying $L$ defined on $\left\{|\arg z|<\eta,|z|>N_{0}\right\}$, where $N_{0}>0$ and $0<\eta<\min \{(\pi / \lambda), \pi\}$. We associate with this $f$ the maps $\beta_{j}$, sectors $S_{j}(\sigma)$, and shires $\Omega_{j}$ of Section 2, as well as the functions $f_{u}, A_{u}$, and $B_{u}$ of Theorem 4. We fix a compact set $K \subset \mathbb{C}$.

In this section we define certain functions $\zeta_{u}$ which satisfy (as we shall see in the next section) the hypotheses of Theorem 4. We need the following lemma, which is an obvious consequence of Lemma 1(b).

Lemma 3. There exist $v_{0}, N>0$ such that, for each $v \geqq v_{0}$, there is exactly one $x \geqq N$ satisfying $x(d / d x)\left[x^{\lambda} L(x)\right]=v$. If that value of $x$ is denoted by $s_{v}$, the numbers $\left\{s_{v}\right\}_{v} \geqq v_{0}$ satisfy

$$
s_{v} \uparrow \infty \quad(v \rightarrow \infty)
$$

Lemma 4. Suppose $R_{1}=\mathbb{R}^{+}$.

(a) There exists $v_{1} \geqq v_{0}$ such that, for all $v \geqq v_{1}$ and $u \in K$, the equation

$$
A_{u}(z)=v
$$

has a complex solution $z=\psi_{u}(v)$ satisfying

$$
u+\psi_{u}(v)=s_{v}+u \lambda^{-1}+o(1) \quad(v \rightarrow \infty),
$$

uniformly for $u \in K$.

(b) There exist $r_{0}>0$ and $v_{2} \geqq v_{1}$ such that, for each $r \geqq r_{0}$ and each $u \in K$, the set $\{z:|z|=$ $r\} \cap\left\{\psi_{u}(v): v>v_{2}\right\}$ consists of exactly one point $\zeta_{u}(r)$. The functions $\zeta_{u}:\left[r_{0}, \infty\right) \rightarrow \mathbb{C}$ 
satisfy

$$
\zeta_{u}(r) \sim r \quad(r \rightarrow \infty)
$$

uniformly for $u \in K$.

Equation (5.2) is called the "saddle point equation".

Proof of Lemma 4. Put $C_{1}=\max \{|u|: u \in K\}$ and $\phi(z)=f(z) \exp \left\{-z^{\lambda} L(z)\right\}$. We begin the proof of (a) by noting that $L$ and $\phi$ are holomorphic in $\left\{|\arg z|<\eta,|z|>N_{0}\right\}$. Thus, in view of (5.1), we may choose $N>0$ so that, for each $v>N$ and $u \in K$, the function

$$
\begin{aligned}
G_{v, u}(w)= & \left(s_{v}-u+w\right) \frac{\phi^{\prime}\left(s_{v}+w\right)}{\phi\left(s_{v}+w\right)} \\
& +\left(s_{v}-u+w\right)\left\{\lambda\left(s_{v}+w\right)^{\lambda-1} L\left(s_{v}+w\right)\right. \\
& \left.+\left(s_{v}+w\right)^{\lambda} L^{\prime}\left(s_{v}+w\right)\right\}-v
\end{aligned}
$$

is defined and holomorphic, in particular, for $|w| \leqq 2 C_{1} \lambda^{-1}$. From the definition of $\phi$ and (2.8) (with $\beta_{1}(z)=z$ since $R_{1}=\mathbb{R}^{+}$) we obtain

$$
\log |\phi(z)|=o(1) \cdot|z|^{\lambda-1} \cdot L(|z|) \quad(|z| \rightarrow \infty),
$$

uniformly for $|\arg z|<\eta$. Hence, in view of (4.9) of Lemma 2 and Lemma 1(c), the first term on the right-hand side of (5.5) is $o(1) \cdot s_{v}^{\lambda-1} \cdot L\left(s_{v}\right)$. Thus, after applications of (4.1) and (4.2), some multiplication, and the observation that $\lambda s_{v}^{\lambda} L\left(s_{v}\right)+s_{v}^{\lambda+1} L^{\prime}\left(s_{v}\right)-v=0$ (by definition of $s_{v}$ ), we may rewrite (5.5) as

$$
\begin{aligned}
G_{v, u}(w)= & \lambda(\lambda-1) s_{v}^{\lambda-1} L\left(s_{v}\right) w+(-u+w) \lambda s_{v}^{\lambda-1} L\left(s_{v}\right) \\
& +(-u+w) L^{\prime}\left(s_{v}\right) s_{v}^{\lambda}+o(1) \cdot s_{v}^{\lambda-1} \cdot L\left(s_{v}\right) \quad(v \rightarrow \infty),
\end{aligned}
$$

uniformly for $u \in K$ and $|w| \leqq 2 C_{1} \lambda^{-1}$. Simplifying and applying (2.1),

$$
\frac{G_{v, u}(w)}{\lambda^{2} s_{v}^{\lambda-1} L\left(s_{v}\right)}=w-u \lambda^{-1}+o(1) \quad(v \rightarrow \infty)
$$

uniformly in $u$ and $w$. An application of Rouchés Theorem reveals the existence of an $N>0$ such that, for each $v>N$ and $u \in K$, the equation $G_{v, u}(w)=0$ has exactly one root $w=w_{u}(v)$ in the disc $D\left(0 ; 2 C_{1} \lambda^{-1}\right)$. Letting $w=w_{u}(v)$ in (5.6), we obtain

$$
w_{u}(v)=u \lambda^{-1}+o(1) \quad(v \rightarrow \infty),
$$

uniformly for $u \in K$. If we now set

$$
\psi_{u}(v)=s_{v}-u+w_{u}(v) \quad(u \in K, v>N)
$$


it follows from (4.12), (4.11), the definition of $\phi$, and the nature of $w_{u}(v)$, that $\psi_{u}(v)$ has the desired properties.

We turn to the proof of (b). Using the definition of $\phi,(4.11),(4.12)$, and (4.13), we express $B_{u}(z)$ (for $|\arg z|<\eta$ ) in terms of $L$ and $\phi$. A short calculation involving (2.1), (4.4), Lemma 2, and Lemma 1(c), then shows that the relation

$$
B_{u}(z) \sim \lambda^{2} z^{\lambda} L(z) \quad(|z| \rightarrow \infty)
$$

holds uniformly for $|\arg z|<\frac{1}{4} \eta$ and $u \in K$. Hence, from (5.3) and Lemma 1(c),

$$
B_{u}\left(\psi_{u}(v)\right) \sim \lambda^{2} s_{v}^{\lambda} L\left(s_{v}\right) \quad(v \rightarrow \infty),
$$

uniformly for $u \in K$. It follows from (5.9) and (4.13) that there is an $N>0$ such that if $v>N$ and $u \in K$, then $A_{u}^{\prime}\left(\psi_{u}(v)\right) \neq 0$. Fix $v>N$ and $u \in K$. Applying the complex implicit function theorem $\left[17\right.$, p. 170] to the function $(w, z) \rightarrow A_{u}(z)-w$ in a neighbourhood of $\left(v, \psi_{u}(v)\right)$, we conclude that $\psi_{u}^{\prime}$ exists in a (real) neighbourhood of $v$. Differentiating the equation $A_{u}\left(\psi_{u}(v)\right)=v$, we see after some manipulation that

$$
\frac{d}{d v} \log \left|\psi_{u}(v)\right|=\operatorname{re}\left\{\frac{1}{B_{u}\left(\psi_{u}(v)\right)}\right\} .
$$

Thus, allowing $v$ to vary and applying (5.9), we have

$$
\frac{d}{d v} \log \left|\psi_{u}(v)\right| \sim \frac{1}{\lambda^{2} s_{v} L\left(s_{v}\right)} \quad(v \rightarrow \infty)
$$

In particular, there is a $v_{2} \geqq v_{1}$ such that for all $u \in K$ and all $v \geqq v_{2},\left|\psi_{u}\right|$ is a continuous and increasing function of $v$. In addition, in view of (5.3), we may assume that $v_{2}$ is so large that, for some $C>0$,

$$
\left|\psi_{u}(v)-s_{v}\right|<C \quad\left(u \in K, v \geqq v_{2}\right) .
$$

Furthermore, again by (5.3),

$$
\left|\psi_{u}(v)\right| \rightarrow \infty \quad(v \rightarrow \infty),
$$

uniformly for $u \in K$. We now choose $r_{0}>\max \left\{\left|\psi_{u}\left(v_{2}\right)\right|: u \in K\right\}$, and the existence and uniqueness of $\zeta_{u}(r)$ follow from the above facts about $\left|\psi_{u}\right|$.

It remains to verify (5.4). For each $u \in K$ and $r \geqq r_{0}$, let $v(u, r)$ be the unique number $v \geqq v_{2}$ such that $\left|\psi_{u}(v)\right|=r$. It is clear from (5.10) and the definition of $\zeta_{u}(r)$ that

$$
\left|\zeta_{u}(r)-s_{v(u, r)}\right|<C \quad\left(u \in K, r>r_{0}\right) .
$$

Since $\zeta_{u}(r)$ lies on the circle $|z|=r$ and $s_{v(u, r)}$ lies on $\mathbb{P}^{+}$, (5.11) implies that $\left|\sin \arg \zeta_{u}(r)\right|<(C / r)\left(u \in K, r>r_{0}\right)$. This gives (5.4), and the lemma. 


\section{Bounds on $f^{(k)}$}

We continue our investigation of the function $f$ fixed in the previous section. The analysis is similar to that carried out by McLeod in [14].

To estimate $f^{(k)}$, we need two lemmas.

Lemima 5. Suppose $R_{1}=\mathbb{R}^{+}$. Fix $\varepsilon, 0<\varepsilon<\eta$. If $J>1$, then

$$
\log \left|\frac{f_{u}\left(r e^{i \theta}\right)}{f_{u}(r)}\right| \leqq \lambda r^{\lambda-1} L(r) \cdot\left\{o(1)+\max _{j>1} \operatorname{re}\left[\beta_{j}(u)-u\right]\right\} \quad(r \rightarrow \infty),
$$

uniformly for $u \in K$ and $\pi \geqq|\theta|>\varepsilon$. If $J=1$, then there exist $N, C>0$ such that if $r>N$, $\pi \geqq|\theta|>\varepsilon$, and $u \in K$, then

$$
\log \left|\frac{f_{u}\left(r e^{i \theta}\right)}{f_{u}(r)}\right| \leqq-C r^{\lambda} L(r)
$$

Proof. Choose $j, 1 \leqq j \leqq J$. By (2.8) and Lemma 1(c),

$$
\log \left|f\left(u+r e^{i \theta}\right) \exp \left\{-\left[\beta_{j}\left(u+r e^{i \theta}\right)\right]^{\lambda} L\left[\beta_{j}\left(u+r e^{i \theta}\right)\right]\right\}\right|=o(1) \cdot r^{\lambda-1} L(r) \quad(r \rightarrow \infty),
$$

uniformly for $u$ and $\theta$ such that $u \in K$ and $u+r e^{i \theta} \in S_{j}(\eta)$. Thus, in view of the definitions (4.11) and (2.4) of $f_{u}$ and $\beta_{j}$,

$$
\log \left|f_{u}\left(r e^{i \theta}\right)\right|=o(1) \cdot r^{\lambda-1} L(r)+\operatorname{re}\left\{\left[r e^{i\left(\theta-\theta_{j}\right)}+\beta_{j}(u)\right]^{\lambda} \cdot L\left[r e^{i\left(\theta-\theta_{j}\right)}+\beta_{j}(u)\right]\right\}
$$

as $r \rightarrow \infty$, uniformly in $u$ and $\theta$ as above. From (6.1) and Lemma 1(c),

$$
\log \left|f_{u}\left(r e^{i \theta}\right)\right|=r^{\lambda} L(r)\left[\cos \lambda\left(\theta-\theta_{j}\right)+o(1)\right] \quad(r \rightarrow \infty),
$$

uniformly in $u$ and $\theta$.

Let $\sigma_{1}$ be as in Lemma $1(\mathrm{f})$, and pick $\delta, 0<\delta<\min \left\{\sigma_{1}, \frac{1}{2} \eta\right\}$. Combining (6.2), the inequality $\eta<\pi / \lambda$ from the definition of maximal rays, (2.9), and Lemma 1 (c), we deduce the existence of numbers $\tau<1$ and $N>0$ such that if $r>N, u \in K$, and $u+r e^{i \theta} \in \mathbb{C}-\bigcup_{q=1}^{J} S_{q}(\delta)$, then

$$
\log \left|f_{u}\left(r e^{i \ell}\right)\right|<\tau r^{\lambda} L(r) .
$$

Meanwhile, returning to (6.1) and using first (4.1) with $w=\beta_{j}(u)$, then (4.3) with $\theta$ replaced by $\theta-\theta_{j}$,

$$
\begin{aligned}
\log \left|f_{u}\left(r e^{i \theta}\right)\right|= & \operatorname{re}\left\{r^{\lambda} e^{i \lambda\left(\theta-\theta_{j}\right)} L\left(r e^{i\left(\theta-\theta_{j}\right)}\right)\right\} \\
& +\lambda r^{\lambda-1} L(r) \operatorname{re}\left\{e^{i(\lambda-1)\left(\theta-\theta_{j}\right)} \beta_{j}(u)+o(1)\right\}
\end{aligned}
$$

as $r \rightarrow \infty$, uniformly for $u$ and $\theta$ such that $u \in K$ and $u+r e^{i \theta} \in S_{j}(\delta)$. In particular, putting 
$\theta=0$ and $j=1$ (whence $\theta_{j}=0$ and $\beta_{j}(u)=u$ ),

$$
\log \left|f_{u}(r)\right|=r^{\lambda} L(r)+\lambda r^{\lambda-1} L(r) \operatorname{re}\{u+o(1)\}
$$

as $r \rightarrow \infty$, uniformly for $u \in K$. Again, from (6.4) and Lemma 1(f),

$$
\log \left|f_{u}\left(r e^{i \theta}\right)\right| \leqq r^{\lambda} L(r)+\lambda r^{\lambda-1} L(r) \operatorname{re}\left\{e^{i(\lambda-1)\left(\theta-\theta_{j}\right)} \beta_{j}(u)+o(1)\right\}
$$

as $r \rightarrow \infty$, uniformly for $u$ and $\theta$ such that $u \in K$ and $u+r e^{i \theta} \in S_{j}(\delta)$.

It is now easy, using (6.6), (6.5), and (6.3), to complete the proof.

Lemma 6. Suppose $R_{1}=\mathbb{R}^{+}$, and define $\psi_{u}(v)$ as in Lemma 4. Then

$$
\log \left|\frac{f\left(u+\psi_{u}(v)\right)}{\left[\psi_{u}(v)\right]^{v}}\right|=s_{v}^{\lambda} L\left(s_{v}\right)-v \log s_{v}+\frac{v}{s_{v}} \operatorname{re} u+o\left(\frac{v}{s_{v}}\right) \quad(v \rightarrow \infty)
$$

uniformly for $u \in K$.

Proof. Note first that, in view of (4.6) and the definition of $s_{v}$ (Lemma 3),

$$
\frac{v}{s_{v}} \sim \lambda s_{v}^{\lambda-1} L\left(s_{v}\right) \quad(v \rightarrow \infty)
$$

Thus, noting that $\beta_{1}(z)=z$ since $R_{1}=\mathbb{R}^{+}$, we have by (2.8), the approximation (5.3) for $u+\psi_{u}(v)$, and Lemma $1(\mathrm{c})$,

$$
\log \mid f\left(u+\psi_{u}(v)\right) \exp \left\{-\left[u+\psi_{u}(v)\right]^{\lambda} L\left[u+\psi_{u}(v)\right]\right\}=o\left(v s_{v}^{-1}\right) \quad(v \rightarrow \infty),
$$

uniformly for $u \in K$.

Next, using (5.3) and (4.1),

$$
\left[u+\psi_{u}(v)\right]^{\lambda} L\left[u+\psi_{u}(v)\right]=s_{v}^{\lambda} L\left(s_{v}\right)+s_{v}^{\lambda-1} L\left(s_{v}\right)(u+o(1)) \quad(v \rightarrow \infty),
$$

uniformly for $u \in K$.

Finally, (5.3) and the Taylor Series for $\log (1+\cdot)$ give

$$
\log \psi_{u}(v)=\log s_{v}+u\left(\lambda^{-1}-1\right) s_{v}^{-1}+o\left(s_{v}^{-1}\right) \quad(v \rightarrow \infty),
$$

uniformly for $u \in K$.

Lemma 6 follows readily from (6.8), (6.9), (6.7), and (6.10). 
Theorem 5. Fix $j, 1 \leqq j \leqq J$.

(a) If $K \cong \Omega_{j}$ and $\lambda>1$, then

$$
\log \left|\frac{f^{(k)}(u)}{k !}\right|=s_{k}^{\lambda} L\left(s_{k}\right)-k \log s_{k}+k s_{k}^{-1} \operatorname{re} \beta_{j}(u)+o\left(k s_{k}^{-1}\right) \quad(k \rightarrow \infty),
$$

uniformly for $u \in K$.

(b) If $K \cong \bar{\Omega}_{j}$ and either $\lambda>1$ or $J=1$, then

$$
\log \left|\frac{f^{(k)}(u)}{k !}\right| \leqq s_{k}^{\lambda} L\left(s_{k}\right)-k \log s_{k}+k s_{k}^{-1} \operatorname{re} \beta_{j}(u)+o\left(k s_{k}^{-1}\right) \quad(k \rightarrow \infty),
$$

uniformly for $u \in K$.

Proof. We assume without loss of generality that $j=1$ and $R_{1}=\mathbb{R}^{+}$. To prove (a), we first verify the hypotheses of Theorem 4 , with $\zeta_{u}(r)$ as in Lemma 4, and with $\Delta_{u}(r)=$ $\left[r^{\lambda} L(r)\right]^{-2 / 5}$ for all $u \in K$. Recall that $\left|\zeta_{u}(r)\right|=r$. Hence, in view of (5.11) and the monotonicity (5.1) of $s_{v}$,

$$
v(u, r) \rightarrow \infty \quad(r \rightarrow \infty),
$$

uniformly for $u \in K$. Thus, by definition of $\zeta_{u}$ and $\psi_{u}$ (Lemma 4), there is an $N>0$ such that

$$
\operatorname{im} A_{u}\left(\zeta_{u}(r)\right)=0 \quad(r>N, u \in K)
$$

Relations (4.14), (4.15), and (4.16) follow instantly from (6.11), (5.8), and (5.4).

We now verify (4.17). Set $\rho=\min \left\{1, \frac{1}{8} \eta\right\}$. From (5.8), Lemma $1(\mathrm{c})$, and the equality $\left|\zeta_{u}(r)\right|=r$, we find that there is an $N>0$ such that, if $z \in D\left(\zeta_{u}(r), \rho r\right)$, then

$$
\left|\frac{B_{u}(z)}{B_{u}\left(\zeta_{u}(r)\right)}\right|<2(1+\rho)^{\lambda} \quad(r>N, u \in K) .
$$

Furthermore, by Lemma 1(b) and the definition $\Delta_{u}(r)=\left[r^{\lambda} L(r)\right]^{-2 / 5}$, there is an $N^{\prime}>0$ such that

$$
\Delta_{u}(r) \leqq \frac{1}{2} \rho \quad(u \in K, r>N) .
$$

We may thus apply Lemma 2 of [14], with $C=2(1+\rho)^{\lambda}, a_{f}=A_{u}, b_{f}=B_{u}$, and $w=\zeta_{u}(r)$, to obtain

$$
\log f_{u}\left(\zeta_{u}(r) e^{i t}\right)=\log f_{u}\left(\zeta_{u}(r)\right)+i t A_{u}\left(\zeta_{u}(r)\right)-\frac{t^{2}}{2} B_{u}\left(\zeta_{u}(r)\right)+E\left(\zeta_{u}(r), t\right)
$$


where

$$
\left|E\left(\zeta_{u}(r), t\right)\right|<\frac{2(1+\rho)^{\lambda}}{\rho}\left|B_{u}\left(\zeta_{u}(r)\right) t^{3}\right| \quad\left(u \in K, r>N^{\prime},|t| \leqq \Delta_{u}(r)\right)
$$

Thus, in view of (5.8) and (5.4),

$$
E\left(\zeta_{u}(r), t\right) \rightarrow 0 \quad(r \rightarrow \infty),
$$

uniformly for $u \in K$ and $|t| \leqq \Delta_{u}(r)$. The formula (4.17) follows from the latter relation along with (6.12) and (6.13).

It remains to verify (4.18). Recall from Section 2 that $f$ has at most finitely many zeros in $|\arg z|<\eta$. Choose $\sigma, \quad 0<\sigma<\min \left\{\frac{1}{8} \eta,(\pi / 3)\right\}$, and expand the function $t \rightarrow \log \left|f_{u}\left(\zeta_{u}(r) e^{i t}\right)\right|$ in a Taylor Series: there exists $N>0$ such that for each $r>N, u \in K$, and $t,|t| \leqq \sigma$, there is a $\xi$ between 0 and $t$ satisfying

$$
\log \left|f_{u}\left(\zeta_{u}(r) e^{i t}\right)\right|=\log \left|f_{u}\left(\zeta_{u}(r)\right)\right|-\operatorname{im} A_{u}\left(\zeta_{u}(r)\right) t-\frac{1}{2} \operatorname{re} B_{u}\left(\zeta_{u}(r) e^{i \xi}\right) t^{2} .
$$

By the latter, along with (6.11), (5.8), (5.4), and the inequality $\left|e^{i \xi}-1\right| \leqq|\xi|$, there is an $N>0$ such that

$$
\log \left|\frac{f_{u}\left(\zeta_{u}(r) e^{i r}\right)}{f_{u}\left(\zeta_{u}(r)\right)}\right| \leqq-\frac{1}{8} \lambda^{2} r^{\lambda} L(r) t^{2} \quad(r>N, u \in K,|t| \leqq \sigma)
$$

It follows from (6.14) and Lemma 1(b) that (4.18) holds under the restriction $\Delta_{u}(r) \leqq|t|<\sigma$.

Again from (6.14) (along with (5.4)), there is an $N>0$ such that

$$
\left|f_{u}(r)\right| \leqq\left|f_{u}\left(\zeta_{u}(r)\right)\right| \quad(r>N, u \in K) .
$$

Up to now, $K$ could have been any compact set. Now, however, we make use of the assumption $K \subset \Omega_{1}$. It follows from Lemma 5 and the definition (2.7) of $\Omega_{1}$ that, if $\lambda>1$ (or even if $\lambda \leqq 1$ and $J=1$ ), then there exist $N, \alpha>0$ such that for all $r>N, u \in K$, and $\theta$, $\pi \geqq|\theta|>\frac{1}{2} \sigma$,

$$
\log \left|\frac{f_{u}\left(r e^{i \theta}\right)}{f_{u}(r)}\right|<-r^{\alpha} L(r)
$$

Thus, in view of (6.15), the relation (4.18) holds under the restriction $\pi \geqq|t| \geqq \sigma$.

We have now verified the hypotheses of Theorem 4. Allowing $\zeta_{u}(r)$ to pass through the values $\psi_{u}(k)\left(k \in \mathbb{Z}^{+}\right)$in the conclusion (4.19), and recalling that $A_{u}\left(\psi_{u}(k)\right)=k$, we see that, if $\lambda>1$ or $J=1$, then

$$
\frac{f^{(k)}(u)}{k !} \sim \frac{f_{u}\left(\psi_{u}(k)\right)}{\left[2 \pi B_{u}\left(\psi_{u}(k)\right)\right]^{1 / 2}\left[\psi_{u}(k)\right]^{k}}
$$


as $k \rightarrow \infty$, uniformly for $u \in K$. Now (5.9), (6.7), Lemma $1(a)$, and $\lambda>1$ imply that

$$
\log \left|2 \pi B_{u}\left(\psi_{u}(k)\right)\right|=\log (2 \pi \lambda k)+o(1)=o\left(\frac{k}{s_{k}}\right) \quad(k \rightarrow \infty),
$$

uniformly for $u \in K$, and Theorem 5(a) follows from (6.16), (6.17), and Lemma 6 .

In the proof of (b), we first use Lemma 5 , the definition (2.7) of $\Omega_{1}$, and (6.15), along with (6.14), to see that

$$
\max \left\{\log \left|\frac{f_{u}\left(\zeta_{u}(r) e^{i t}\right)}{f_{u}\left(\zeta_{u}(r)\right)}\right|:-\pi<t \leqq \pi\right\} \leqq o(1) \cdot r^{\lambda-1} L(r)
$$

as $r \rightarrow \infty$, uniformly for $u \in K$. Introduction of the factor $\left|f_{u}\left(\zeta_{u}(r)\right)\right|$ into Cauchy's Estimates then yields

$$
\log \left|\frac{f_{u}^{(k)}(0)}{k !}\right| \leqq o(1) \cdot r^{\lambda-1} L(r)+\log \left|\frac{f_{u}\left(\zeta_{u}(r)\right)}{r^{k}}\right| \quad(r \rightarrow \infty),
$$

uniformly for $k \in \mathbb{Z}^{+}$and $u \in K$. Taking $r=\left|\psi_{u}(k)\right|$ in (6.18), Theorem $5(\mathrm{~b})$ is easily derived from Lemma 6. This completes the proof of Theorem 5.

We need in the sequel the following two relations. First, let $0<C_{1}<C_{2}$. A calculation involving Stirling's formula and the fact (from 6.7)) that $s_{k}^{\lambda} L\left(s_{k}\right) \sim k / \lambda$ shows that

$$
k ! \exp \left\{s_{k}^{\lambda} L\left(s_{k}\right)-k \log s_{k}+x \frac{k}{s_{k}}\right\}=\left[\frac{k}{s_{k}} e^{1 / \lambda-1}(1+o(1))\right]^{k} \quad(k \rightarrow \infty),
$$

uniformly in $x, C_{1}<x<C_{2}$. Secondly, by (6.7) and Lemma 1(a),

$$
\frac{k}{s_{k}} \rightarrow \begin{cases}\infty & (\text { if } \lambda>1) \\ 0 & (\text { if } \lambda<1)\end{cases}
$$

as $k \rightarrow \infty$.

Corollary. If $\lambda>1$ then, for each $a \in \mathbb{C}$, the final set $\Lambda(a, f)$ is contained in $\bigcup_{j=1}^{J} \partial \Omega_{j}$.

Proof. It is clear from the definition of the shires that every point in the plane is contained in the closure of some $\Omega_{j}$. Hence it is enough to show that $\Lambda(a, f) \subset \mathbb{C}-$ $\bigcup_{j=1}^{J} \Omega_{j}$. Fix $j$ and $z \in \Omega_{j}$, and choose $\rho>0$ so that $\overline{D(z ; \rho)} \subset \Omega_{j}$. Then, by Theorem 5 , (6.19), and (6.20),

$$
\left|f^{(k)}(u)\right| \rightarrow \infty \quad(k \rightarrow \infty)
$$

uniformly for $u \in \overline{D(z ; \rho)}$. The desired inclusion now follows from the definition of final set. 


\section{Proofs of the main theorems}

We now prove Theorems 1,2 , and 3. In each proof we assume that the function $f$ of the theorem is the function we fixed in Section 5 .

Proof of Theorem 3. First suppose $\lambda<1$. It is clear from Theorem 5(b), (6.19), and (6.20), that

$$
\left|f^{(k)}(u)\right| \rightarrow 0 \quad(k \rightarrow \infty),
$$

uniformly for $u$ in compact subsets of the plane. Hence, if $a \neq 0$, then $\Lambda(a, f)$ is void. If we now suppose that $\lambda \leqq 1$, then (6.16) and the fact that $f$ has at most finitely many zeros in $|\arg z|<\eta$ imply that $\Lambda(0, f)$ is void. This completes the proof.

The following lemma is, with a change in notation, due to Edrei. Its proof is given in [5].

Lemma 7 (Edrei). Suppose $\rho_{1}>0$. Let $\left\{F_{k}\right\}_{k=1}^{\infty}$ be a sequence of functions analytic and not identically zero in the disc $\overline{D\left(0 ; \rho_{1}\right)}$. Put

$$
U_{k}(\rho)=\frac{1}{2 \pi} \int_{0}^{2 \pi} \log \left|F_{k}\left(\rho e^{i \theta}\right)\right| d \theta \quad\left(0<\rho \leqq \rho_{1}\right) .
$$

Let $\left\{b_{k}\right\}_{k=1}^{\infty}$ be a sequence of positive numbers, and assume that

$$
U_{k}(\rho) \sim b_{k} \rho \quad(k \rightarrow \infty),
$$

uniformly for $\frac{1}{4} \rho_{1} \leqq \rho \leqq \rho_{1}$. Then, for each $\sigma, \frac{1}{4} \rho_{1}<\sigma<\rho_{1}$,

$$
n\left(\sigma, 0, F_{k}\right) \sim b_{k} \sigma \quad(k \rightarrow \infty) .
$$

In the proof of Theorem 1 we estimate the number of a-points near points on the boundaries of the shires. The method is that used by Edrei [5].

Proof of Theorem 1. In view of the Corollary (Section 6), it is enough to show that if $z \in \bigcup_{j=1}^{j} \partial \Omega_{j}$, then $z \in \Lambda(a, f)$. We assume without loss of generality that $z=0$, and that there is a $J^{\prime}, 1 \leqq J^{\prime} \leqq J$, such that $0 \in \partial \Omega_{j}$ if and only if $j \leqq J^{\prime}$. (Then $J^{\prime} \geqq 2$.) We assume also that $\theta_{1}<\theta_{2}<\cdots<\theta_{J}$.

Put $\theta_{0}=\theta_{\boldsymbol{J}},-2 \pi$, and

$$
\begin{gathered}
\omega_{j}=\frac{\theta_{j}+\theta_{j-1}}{2} \quad\left(j=1, \ldots, J^{\prime}\right) \\
\omega_{J^{\prime}+1}=\omega_{1}+2 \pi=\frac{\left(\theta_{1}+2 \pi\right)+\theta_{J^{\prime}}}{2} .
\end{gathered}
$$


Then the numbers $\omega_{1}, \ldots, \omega_{J^{\prime}}, \omega_{J^{\prime}+1}$ are, respectively, the midpoints of the intervals $\left(\theta_{0}, \theta_{1}\right), \ldots,\left(\theta_{J^{\prime}-1}, \theta_{J^{\prime}}\right),\left(\theta_{J^{\prime}}, \theta_{1}+2 \pi\right)$.

Choose $\rho_{0}>0$ so that $\overline{D\left(0 ; 5 \rho_{0}\right)} \subset \bigcup_{j=1}^{J^{\prime}} \bar{\Omega}_{j}$. (This is possible for the following reason. By definition of shire ((2.7) and (2.4)), every point in $\mathbb{C}$ is in the closure of some shire. Thus, if $\rho_{0}$ does not exist, then there is a shire $\Omega_{q}, q>J^{\prime}$, such that every disc $D(0 ; \rho)$ contains some point of $\overline{\boldsymbol{\Omega}}_{q}$. Then $z \in \partial \boldsymbol{\Omega}_{q}$, contrary to hypothesis.) For $j, \rho$, and $\theta$ such that $1 \leqq j \leqq J^{\prime}, 0<\rho<4 \rho_{0}$, and $\omega_{1} \leqq \theta \leqq \omega_{1}+2 \pi$, we see from the definition (2.4) of $\beta_{j}$ and the hypothesis $0 € \partial \Omega_{j}$ that

$$
\operatorname{re} \beta_{j}\left(\rho e^{i \theta}\right)=\operatorname{re} \beta_{1}(0)+\rho \cdot \operatorname{re} e^{i\left(\theta-\theta_{j}\right)} .
$$

Thus, by the definition (2.7) of shire, if $0<\rho<4 \rho_{0}$, then

$$
\rho e^{i \theta} \in \Omega_{j} \quad \text { if and only if } \omega_{j}<\theta<\omega_{j+1} .
$$

Fix $a \in \mathbb{C}$. For $u \in \mathbb{C}$ and integers $k>v_{0}$, put

$$
F_{k}(u)=\frac{f^{(k)}(u)-a}{k !} \cdot \exp \left\{-s_{k}^{\lambda} L\left(s_{k}\right)+k \log s_{k}-\operatorname{re}\left[\beta_{1}(0)\right] \frac{k}{s_{k}}\right\}
$$

Then the zeros of $F_{k}$ and $f^{(k)}-a$ coincide and have the same multiplicities.

We claim that the formulas of Theorem 5 remain valid if $f^{(k)}(u)$ is replaced by $f^{(k)}(u)$ $-a$. To prove the claim, say, for the first formula, we exponentiate the formula, multiply by $k$ !, and, with the aid of (6.19), (6.20), and the inequality $\lambda>1$, obtain

$$
\begin{aligned}
\left|f^{(k)}(u)-a\right|= & k ! \exp \left\{s_{k}^{\lambda} L\left(s_{k}\right)-k \log s_{k}\right. \\
& \left.+k s_{k}^{-1} \mathrm{re} \beta_{j}(u)+o\left(k s_{k}^{-1}\right)\right\}(1+o(1)) \quad(k \rightarrow \infty) .
\end{aligned}
$$

The claim follows upon rewriting the factor $1+o(1)$ as $\exp \left\{o\left(k s_{k}^{-1}\right)\right\}$, as we may do since, by $(6.20), k s_{k}^{-1} \rightarrow \infty$.

From Theorem 5 (with $f^{(k)}(u)$ replaced by $\left.f^{(k)}(u)-a\right)$, (7.3), (7.4), and the equations re $\beta_{j}(0)=\operatorname{re} \beta_{1}(0)\left(j=1, \ldots, J^{\prime}\right)$, we obtain the following formulas, valid for each fixed $\tau, \delta$, and $j, 0<\tau<4 \rho_{0}, 0<\delta<\frac{1}{2} \min _{j}\left\{\omega_{j+1}-\omega_{j}\right\}, 1 \leqq j \leqq J^{\prime}$ :

$$
\log \left|F_{k}\left(\rho e^{i \theta}\right)\right|=\frac{k}{s_{k}} \rho \cos \left(\theta-\theta_{j}\right)+o\left(\frac{k}{s_{k}}\right) \quad(k \rightarrow \infty),
$$

uniformly in $\rho, \tau \leqq \rho \leqq 4 \rho_{0}$, and $\theta, \omega_{j}+\delta \leqq \theta \leqq \omega_{j+1}-\delta$;

$$
\log \left|F_{k}\left(\rho e^{i \theta}\right)\right| \leqq \frac{k}{s_{k}} \rho \cos \left(\theta-\theta_{j}\right)+o\left(\frac{k}{s_{k}}\right) \quad(k \rightarrow \infty),
$$

uniformly in $\rho, 0 \leqq \rho \leqq 4 \rho_{0}$, and $\theta, \omega_{j} \leqq \theta \leqq \omega_{j+1}$. 
For $\delta$ as above, put

$$
I_{\delta}=\left[\omega_{1}, \omega_{1}+\delta\right] \cup \bigcup_{j=2}^{J^{\prime}}\left[\omega_{j}-\delta, \omega_{j}+\delta\right] \cup\left[\omega_{J^{\prime}+1}-\delta, \omega_{J^{\prime}+1}\right]
$$

Also put $I=\left[\omega_{1}, \omega_{J^{\prime}+1}\right]$ and

$$
Q=\frac{1}{\pi} \sum_{j=1}^{J^{\prime}} \sin \left(\frac{\theta_{j}-\theta_{j-1}}{2}\right)
$$

Then $Q>0$. Upon integrating (7.5) and (7.6), we deduce from the definition (7.1) of $\omega_{j}$ that, if $0<\tau<4 \rho_{0}$, then

$$
\frac{1}{2 \pi} \int_{I} \log \left|F_{k}\left(\rho e^{i \theta}\right)\right| d \theta \leqq Q \frac{k}{s_{k}} \rho(1+o(1)) \quad(k \rightarrow \infty),
$$

and

$$
\frac{1}{2 \pi} \int_{I-I_{\delta}} \log \left|F_{k}\left(\rho e^{i \theta}\right)\right| d \theta=Q \frac{k}{s_{k}} \rho(1+o(1)) \quad(k \rightarrow \infty, \delta \rightarrow 0),
$$

both uniformly in $\rho, \tau \leqq \rho \leqq 4 \rho_{0}$.

Fix $\tau, 0<\tau<\rho_{0}$. We will show that

$$
\int_{I_{\delta}} \log ^{+}\left|\frac{1}{F_{k}\left(\rho e^{i \theta}\right)}\right| d \theta=o(1) \cdot \frac{k}{s_{k}} \rho \quad(k \rightarrow \infty, \delta \rightarrow 0),
$$

uniformly in $\rho, \tau \leqq \rho \leqq \rho_{0}$. To this end we need the Poisson-Jensen Formula [9, p. 1],

$$
\begin{aligned}
\log \left|F_{k}(u)\right|= & \frac{1}{2 \pi} \int_{0}^{2 \pi} \log \left|F_{k}\left(r e^{i \Phi}\right)\right| \cdot P(r,|u|, \Phi-\theta) d \Phi \\
& -\sum_{\left.\right|_{q} \backslash \leqq r} \log \left|\frac{r^{2}-d_{q} u}{r\left(u-d_{q}\right)}\right| \quad\left(u=|u| e^{i \theta},|u|<r\right),
\end{aligned}
$$

where $\left\{d_{q}\right\}(q=1,2,3, \ldots)$ are the zeros of $F_{k}$, and

$$
P(r,|u|, \Phi-\theta)=\frac{r^{2}-|u|^{2}}{r^{2}-2 r|u| \cos (\Phi-\theta)+|u|^{2}}
$$

is the Poisson Kernel. We need also the standard inequality

$$
\frac{r-|u|}{r+|u|} \leqq P(r,|u|, \Phi-\theta) \leqq \frac{r+|u|}{r-|u|},
$$


and the identity

$$
\log x=\log ^{+} x-\log ^{+} \frac{1}{x} \quad(x>0)
$$

We now begin the proof of (7.11).

Relations (7.5) and (6.20) imply that there is an $N>0$ such that

$$
\log \left|F_{k}\left(\rho_{0} e^{i \theta_{1}}\right)\right|>0 \quad(k>N) .
$$

Put $u=\rho_{0} e^{i \theta_{1}}$ and $r=2 \rho_{0}$ in (7.12). The positivity of each term of $\sum \log |\cdot|$ in (7.12), together with (7.15), (7.14), and (7.13), imply that

$$
m\left(2 \rho_{0}, \frac{1}{F_{k}}\right)<9 m\left(2 \rho_{0}, F_{k}\right) \quad(k>N) .
$$

If $\left|d_{q}\right| \leqq 2 \rho_{0}$, then, by the triangle inequality,

$$
\left|\frac{\left(4 \rho_{0}\right)^{2}-d_{q} \rho_{0} e^{i \theta_{1}}}{4 \rho_{0}\left(\rho_{0} e^{i \theta_{1}}-d_{q}\right)}\right|>\frac{7}{6}
$$

Setting $r=4 \rho_{0}$ and $u=\rho_{0} e^{i \theta_{1}}$ in (7.12), neglecting some terms of $\sum \log |\cdot|$, and using (7.17), (7.14), and (7.13), we obtain

$$
\left(\log \frac{7}{6}\right) n\left(2 \rho_{0}, 0, F_{k}\right) \leqq-\log \left|F_{k}\left(\rho_{0} e^{i \theta_{1}}\right)\right|+2 m\left(4 \rho_{0}, F_{k}\right)
$$

so that, by (7.15) and (7.6), there exist $C, N>0$ such that

$$
n\left(2 \rho_{0}, 0, F_{k}\right) \leqq C \frac{k}{s_{k}} \quad(k>N) .
$$

Reversing the sign of (7.12), setting $r=2 \rho_{0}$ and $u=\rho e^{i \theta}$, and recalling that $\rho \leqq \rho_{0}$, we deduce that

$$
\log ^{+}\left|\frac{1}{F_{k}\left(\rho e^{i \theta}\right)}\right| \leqq 3 m\left(2 \rho_{0}, \frac{1}{F_{k}}\right)+\sum_{\mid d q} \sum_{\mid \leqq 2 \rho_{0}} \log \left|\frac{3 \rho_{0}}{\rho e^{i \theta}-d_{q}}\right| .
$$

Fix $q$ and write $d_{q}=\left|d_{q}\right| e^{i \gamma}$. Clearly $\left|\rho e^{i \theta}-d_{q}\right|>\left|\operatorname{im}\left[\left(\rho e^{i \theta}-d_{q}\right) e^{-i(\gamma+\theta) / 2}\right]\right|$, whence

$$
\left|\rho e^{i \theta}-d_{q}\right|>\rho\left|\sin \left(\frac{\theta-\gamma}{2}\right)\right| \text {. }
$$


An examination of the graph of

$$
x \rightarrow \log \left(\frac{1}{|\sin (x / 2)|}\right)
$$

shows that, in view of the definition (7.7) of $I_{\delta}$,

$$
\int_{I_{\delta}} \log \left|\frac{1}{\sin ((\theta-\gamma) / 2)}\right| d \theta \leqq 2 J^{\prime} \int_{0}^{\delta} \log \left(\frac{1}{\sin (x / 2)}\right) d x .
$$

Hence, in view of the inequality $\sin (z / 2)<(x / \pi)$, there exist $\delta_{0}, C>0$, independent of $q$, such that

$$
\int_{I_{b}} \log \left|\frac{1}{\sin ((\theta-\gamma) / 2)}\right| d \theta<C \delta \log \delta \quad\left(\delta<\delta_{0}\right) .
$$

We conclude from (7.19), (7.20), and (7.21), that

$$
\int_{I_{\delta}} \log ^{+}\left|\frac{1}{F_{k}\left(\rho e^{i \theta}\right)}\right| d \theta \leqq 6 J^{\prime} \delta m\left(2 \rho_{0}, \frac{1}{F_{k}}\right)+n\left(2 \rho_{0}, 0, F_{k}\right) \cdot C \delta \log \delta .
$$

The desired inequality (7.11) follows from (7.22), (7.16), (7.6), and (7.18).

It is a consequence of (7.9), (7.10), (7.11), and the definition (7.1) of $U_{k}(\rho)$, that

$$
U_{k}(\rho) \sim Q \frac{k}{s_{k}} \rho \quad(k \rightarrow \infty),
$$

uniformly in $\rho, \tau \leqq \rho \leqq \rho_{0}$. Thus, from Lemma 7 and the definition of $F_{k}$,

$$
n\left(\sigma, a, f^{(k)}\right) \sim Q \frac{k}{s_{k}} \sigma \quad(k \rightarrow \infty)
$$

for each $\sigma, 0<\sigma<\rho_{0}$. Theorem 1 follows from (7.23), (6.20), and the positivity of $Q$.

Proof of Theorem 2. In view of (7.23) and the definiton (7.8) of $Q$, it is enough to show that, if $t_{k}$ is given by (3.2), then

$$
\frac{k}{s_{k}} \sim t_{k} \quad(k \rightarrow \infty)
$$

We first show that, if $\gamma$ is real, then

$$
L\left[x(L(x))^{\eta}\right] \sim L(x) \quad(x \rightarrow \infty) .
$$


To see this, we assume without loss of generality that $\mu<1$ and use (3.1) to pick $C$, $N>0$ so that

$$
-\frac{C}{t(\log t)^{\mu}}<\frac{L^{\prime}(t)}{L(t)}<\frac{C}{t(\log t)^{\mu}} \quad(t \geqq N) .
$$

Integration of (7.26) from $N$ to $x$ reveals that

$$
\log L(x)=O(1) \cdot(\log x)^{1-\mu} \quad(x \rightarrow \infty) .
$$

Furthermore, the fundamental theorem of calculus, Lemma 1(a), and (7.26) imply that there is an $N>0$ such that, for $x>N$,

$$
\left|\log L(x)-\log L\left[x(L(x))^{\nu}\right]\right|<\frac{C}{1-\mu}\left|(\log x)^{1-\mu}-[\log x+\gamma \log L(x)]^{1-\mu}\right|
$$

whence, by the binomial theorem and (7.27),

$$
\log \frac{L(x)}{L\left[x(L(x))^{\gamma}\right]}=O(1) \cdot(\log x)^{1-2 \mu} \quad(x \rightarrow \infty) .
$$

Since $\mu>\frac{1}{2},(7.28)$ implies (7.25).

To prove (7.24), we put $H(x)=\lambda x^{\lambda} L(x)$, and observe that, by (4.6) and the definition of $s_{k}$ (Lemma 3),

$$
H\left(s_{k}\right) \sim k \quad(k \rightarrow \infty) .
$$

Meanwhile, if $C>0$, then the definition (3.2) of $t_{k}$, along with Lemma 1(c) and (7.25) (with $z=(k / \lambda)^{1 / \lambda}$ and $\gamma=-(1 / \lambda)$ ) give

$$
H\left(C \frac{k}{t_{k}}\right) \sim C^{\lambda} k \quad(k \rightarrow \infty) .
$$

Fix $\varepsilon>0$. Setting $C=1+\varepsilon$ in (7.30) and using (7.29), we find that there exists $N>0$ such that

$$
H\left((1+\varepsilon) \frac{k}{t_{k}}\right)>H\left(s_{k}\right) \quad(k>N)
$$

But $s_{k} \rightarrow \infty$ and $k / t_{k} \rightarrow \infty$ (by (5.1), (3.2), and Lemma 1(a)), and thus, by (7.31) and the monotonicity of $H$ (Lemma $1(\mathrm{~b}))$, there is an $N^{\prime}>0$ such that

$$
s_{k}<(1+\varepsilon) \frac{k}{t_{k}} \quad\left(k>N^{\prime}\right)
$$


The latter inequality, in view of the arbitrary nature of $\varepsilon$, gives half of (7.24). A similar argument with $C=1-\varepsilon$ in (7.30) gives the other half of (7.24). This completes the proof of Theorem 2.

\section{Examples}

We determine in this section the final sets of some specific functions. Our aim is not to give the most general results possible, but rather to indicate some interesting consequences of the main theorems.

Theorem 8, which appears later in this section, yields the following examples.

Examples. Let $a \in \mathbb{C}$.

(a) If $f_{1}(z)=\cos z^{2}$, then $\Lambda\left(a, f_{1}\right)$ is the union of the real and imaginary axes.

(b) If $f_{2}(z)=\exp \left(z^{2}\right)+\exp \left(-z^{2}\right)+\exp \left(-i z^{2}\right)$, then

$$
\Lambda\left(a, f_{2}\right)=\left\{r e^{i \theta}: r \geqq 0, \theta=\frac{\pi}{8}, \frac{3 \pi}{8}, \frac{6 \pi}{8}, \frac{9 \pi}{8}, \frac{11 \pi}{8}, \frac{15 \pi}{8}\right\} .
$$

(c) If $f_{3}(z)=\exp \left[(z-1)^{3}\right]+\exp \left[-(z+1)^{3}\right]$, then $\Lambda\left(a, f_{3}\right)$ is the union of the imaginary axis, the segment joining -1 to +1 , and the four rays

$$
\left\{1+r e^{ \pm i \pi / 6}: r \geqq 0\right\} \text { and }\left\{-1+r e^{ \pm i 5 \pi / 6}: r \geqq 0\right\} \text {. }
$$

The examples given above differ from those of Pólya and McLeod, in that, in the latter, if $\lambda$ denotes the order of the function, then the final set consists of $\lambda$ equally spaced rays emanating from a single point.

The Mittag-Leffler functions [2, p. 50] are defined, for $\lambda>\frac{1}{2}$, by

$$
E_{\lambda}(z)=\sum_{p=0}^{\infty} \frac{z^{p}}{\Gamma\left(1+p \lambda^{-1}\right)} \quad(z \in \mathbb{C})
$$

In particular, $E_{1}(z)=e^{z}$. The following theorem gives a generalization of $E_{\lambda}$.

For $0<\sigma<\pi$ and $N>0$, put

$$
S_{\sigma, N}=\{|\arg w|<\sigma,|w|>N\} .
$$

Theorem 6. Suppose $\lambda>\frac{1}{2}, N>0$, and $(\pi / 2 \lambda)<\eta<\pi$. Suppose $L$ is slowly varying on $S_{\eta, N}$. Then there exists an entire function $f_{L, \lambda}$ such that, for each $\sigma$ satisfying $(\pi / 2 \lambda)<\sigma<\eta$, there is an $M>0$ such that

$$
\left|f_{L, \lambda}(z)-\lambda \exp \left\{z^{\lambda} L(z)\right\}\right|<M \quad\left(z \in S_{\sigma, N}\right),
$$


whereas

$$
\left|f_{L, \lambda}(z)\right|<M \quad\left(z \in \mathbb{C}-S_{\sigma, N}\right) .
$$

If $\lambda \neq 1$ then, for each $a \in \mathbb{C}, \Lambda\left(a, f_{L, \lambda}\right)$ is void. If $\lambda=1$, then $\Lambda\left(0, f_{L, \lambda}\right)$ is void.

Remark. If $L(z) \equiv 1$, then $f_{L, \lambda}=E_{\lambda}$.

Proof of Theorem 6. Fix $\tau,(\pi / 2 \lambda)<\tau<\eta$. For $r>N$, let $C(r)$ be the contour $\left\{r e^{i \theta}\right.$ : $-\tau \leqq \theta \leqq \tau\}$, oriented counterclockwise, and set

$$
f_{r}(z)=\frac{\lambda}{2 \pi i} \int_{C(r)} \frac{\exp \left\{w^{\lambda} L(w)\right\}}{w-z} d w \quad(z \in \mathbb{C})
$$

It is not difficult to show, with the aid of (4.3) and Lemma 1(b), that $f_{r}$ converges to an entire function $f_{L, \lambda}$ satisfying (8.1) and (8.2). We omit the details, which are similar to those given by Heins $[10$, p. 127] for the case $L(z) \equiv 1$.

It follows from (8.1), (8.2), (4.3), and Lemma $1(\mathrm{~b})$, that $f_{L, \lambda}$ has the single maximal ray $\mathbb{R}^{+}$. The assertions about $\Lambda\left(a, f_{L, \lambda}\right)$ thus follow from Theorems 1 and 3. This completes the proof of Theorem 6 .

It is a consequence of the next theorem that, if $\left\{R_{j}\right\}_{j=1}^{J}$ are rays as in Section 2, then there exists a function having maximal rays $\left\{R_{j}\right\}_{j=1}^{J}$.

Theorem 7. Let $\left\{R_{j}\right\}_{j=1}^{J},\left\{\beta_{j}\right\}_{j=1}^{J}$, and $\left\{\Omega_{j}\right\}_{j=1}^{J}$ be associated rays, maps, and shires as in Section 2. Suppose $\lambda>1, N>0$, and $(\pi / 2 \lambda)<\eta<\pi$. Let $L$ be slowly varying on $S_{\eta, N}$, and let $f_{L, i}$ be as in Theorem 6 . Assume that $c_{1}, \ldots, c_{J}$ are non-zero complex constants. If

$$
f(z)=\sum_{j=1}^{J} c_{j} f_{L, \lambda}\left(\beta_{j}(z)\right) \quad(z \in \mathbb{C}),
$$

then $f$ has maximal rays $\left\{R_{j}\right\}_{j=1}^{J}$ and, for each $a \in \mathbb{C}, \Lambda(a, f)=\bigcup_{j=1}^{J} \partial \Omega_{j}$.

Proof. Define sectors $S_{j}(\cdot)$ by (2.5). Choose $\sigma, 0<\sigma<(\pi / \lambda)$, so small that $S_{j}(\sigma) \cap S_{q}(\sigma)$ is bounded for all pairs of distinct integers $j$ and $q$. From the definition (2.4) of $\beta_{j}$,

$$
\beta_{j}\left(r e^{i \theta}\right)=\beta_{j}(0)+r e^{i\left(\theta-\theta_{j}\right)} \quad(j=1, \ldots, J) .
$$

A calculation involving (8.1), (8.4), and Lemma 1(c) shows that, for each $j$,

$$
\left|f_{L, \lambda}\left(\beta_{j}\left(r e^{i \theta}\right)\right)\right|>\lambda \exp \left\{r^{\lambda} L(r) \cdot\left(\cos \lambda\left(\theta-\theta_{j}\right)+o(1)\right)\right\}-M \quad(r \rightarrow \infty),
$$

uniformly for $\theta$ such that $r e^{i \theta} \in S_{j}(\sigma)$, so that, by (8.3), (8.2), and Lemma 1(b),

$$
f\left(r e^{i \theta}\right) \exp \left\{-\left[\beta_{j}\left(r e^{i \theta}\right)\right]^{\lambda} L\left[\beta_{j}\left(r e^{i \theta}\right)\right]\right\} \rightarrow C_{j} \quad(r \rightarrow \infty),
$$

uniformly for $r e^{i \theta} \in S_{j}(\sigma)$. Similar considerations show that there exist $N>0$ and $\gamma$, 
$0<\gamma<1$, such that, for each $j$,

$$
\left|f_{L, \lambda}\left(\beta_{j}(z)\right)\right|<\exp \left\{\gamma|z|^{\lambda} L(|z|)\right\} \quad\left(|z|>N, z \in \mathbb{C}-\bigcup_{j=1}^{J} S_{j}(\sigma)\right) .
$$

Theorem 7 follows from (8.6), (8.7), the definition of maximal rays, and Theorem 1.

Our next result concerns sums of exponential powers.

Theorem 8. Let $\lambda, M \in \mathbb{Z}^{+}$, and let $\Phi_{1}, \ldots, \Phi_{M}$ be real numbers such that the $\lambda M$ numbers $\Phi_{m}+2 \pi j \lambda^{-1}(m=1, \ldots, M ; j=0, \ldots, \lambda-1)$ are all distinct. Let $v_{1}, \ldots, v_{M} \in \mathbb{C}$. Then the function

$$
f(z)=\sum_{m=1}^{M} \exp \left\{\left[e^{-i \Phi_{m}}\left(z-v_{m}\right)\right]^{\lambda}\right\} \quad(z \in \mathbb{C})
$$

has maximal rays $\left\{R\left(v_{m}, \Phi_{m}+2 \pi j \lambda^{-1}\right)\right\}(m=1, \ldots, M ; j=0, \ldots, \lambda-1)$, and its final sets are given by Theorem 1.

Proof. Let $L(z) \equiv 1$. By (8.1), (8.2), and Liouville's Theorem there are complex numbers $w_{1}, \ldots, w_{M}$ such that

$$
\lambda \cdot \exp \left\{\left[e^{-i \Phi_{m}}\left(z-v_{m}\right)\right]^{\lambda}\right\}=\sum_{j=1}^{\lambda} f_{L, \lambda}\left(e^{-i\left(\Phi_{m}+2 \pi j / \lambda\right)}\left(z-v_{m}\right)\right)+w_{m} \quad(z \in \mathbb{C}) .
$$

Theorem 8 follows from (8.8) and Theorem 7.

The following result is an obvious consequence of Theorems 8 and 2.

Theorem 9. Let $\lambda$ be an integer greater than one. Let $a \in \mathbb{C}$. For $z \in \mathbb{C}$, set $f(z)=\exp \left(z^{\lambda}\right)$, and

$$
h(z)= \begin{cases}\pi^{-1} \lambda^{1+1 / \lambda} \sin (\pi / \lambda) & \text { if } z=0 \\ 2 \pi^{-1} \lambda^{1 / \lambda} \sin (\pi / \lambda) & \text { if } z \neq 0\end{cases}
$$

Then for every $z \in \Lambda(a, f)$, there is a $\rho_{0}>0$ such that for each $\rho, 0<\rho<\rho_{0}$, the number of a-points of $f^{(k)}$ in $D(z ; \rho)$ is asymptotic, as $k \rightarrow \infty$, to $h(z) k^{1-1 / \lambda} \rho$.

We consider finally a subclass of the Lindelöf functions, namely, the subclass

$$
P_{\lambda}(z)=\prod_{1}^{\infty}\left(1+\frac{z}{n^{1 \backslash \lambda}}\right) \quad(z \in \mathbb{C}, 0<\lambda<1) .
$$

Theorem 10. For each $a \in \mathbb{C}$, and each $\lambda, 0<\lambda<1, \Lambda\left(a, P_{\lambda}\right)$ is void.

Proof. We need the following formulas of Lindelöf [12, pp. 49-53], valid for 
$0<\delta<\pi$

$$
\frac{P_{\lambda}^{\prime}(z)}{P_{\lambda}(z)} \sim \frac{\pi \lambda}{\sin \pi \lambda} z^{\lambda-1} \quad(|z| \rightarrow \infty)
$$

and

$$
\log P_{\lambda}(z) \sim \frac{\pi}{\sin \pi \lambda} z^{\lambda} \quad(|z| \rightarrow \infty),
$$

both uniformly for $|\arg z|<\pi-\delta$. We also need a result proved by Hardy [7] and again, recently, by Abi-Khuzam [1]: There exists a $\delta_{0}>0$ such that

$$
P_{\lambda}(z) \sim(2 \pi)^{1 / 2}(-z)^{-1 / 2} 2 \sin \left[\pi(-z)^{\lambda}\right] \cdot \exp \left\{(\pi \cot \pi \lambda)(-z)^{\lambda}\right\} \quad(|z| \rightarrow \infty),
$$

uniformly for $|\arg z-\pi|<\delta_{0}$.

Fix $\lambda$. With the principal branch of the logarithm, set

$$
L(z)=\frac{\log P_{\lambda}(z)}{z^{\lambda}} \quad(|\arg z|<\pi) .
$$

It is clear from (8.9), (8.10), and (8.11) that $L$ is slowly varying. Furthermore, by (8.13), (8.12), and (8.11), $P_{\lambda}$ has the single maximal ray $\mathbb{R}^{+}$relative to $L$ and $\lambda$. Theorem 10 thus follows from Theorem 3. This completes the proof.

I am indebted to Professors A. Baernstein and C. Prather for bringing to my attention, respectively, [7] and [1].

\section{REFERENCES}

1. F. F. Aвi-Kниzам, The asymptotic behavior of a Lindelöf function and its Taylor coefficients, J. of Math. Anal. and Appl. 93 (1983), 495-526.

2. M. L. CARTwright, Integral Functions (Cambridge University Press, 1962).

-3. N. G. DeBruin, Asymptotic Methods in Analysis (North Holland Pub. Co., Amsterdam, 1958).

4. A. EDREI, Zeros of successive derivatives of entire functions of the form $h(z) \exp \left(-e^{z}\right)$, Trans. Amer. Math. Soc. 259 (1980), 207-226.

5. A. EDrei, Zeros of successive derivatives of certain entire functions of infinite order, Functions, series, operators, Vol. I, II (Budapest, 1980), 449-484; Colloq. Math. Soc. János Bolyai 35 (North-Holland, Amsterdam-New York, 1983).

6. R. M. Gethner, A Pólya "shire" theorem for entire functions, (Ph.D. thesis, University of Wisconsin, 1982).

7. G. H. Hardy, On the function $P_{\rho}(x)$, Quart. J. Math. Oxford Ser. (2), 37 (1906), 146-172.

8. W. K. Hayman, A generalisation of Stirling's formula, J. Reine Agnew. Math. 196 (1956), 67-95. 
9. W. K. Hayman, Meromorphic Functions (Clarendon Press, Oxford, 1964).

10. M. H. Herns, Selected Topics in the Theory of Functions of a Complex Variable (Holt, Rinehart, and Winston, New York, 1962).

11. B. JA. Levin, Distribution of Zeros of Entire Functions, Rev. Ed. (Amer. Math. Soc., Providence, 1980).

12. E. LindelöF, Mémoire sur la théorie des fonctions entières de genre fini, Acta Soc. Sci. Fenn. 31 (1901), 1-77.

13. R. M. McLeod, On the zeros of the derivatives of some entire functions (Ph.D. thesis, The Rice Institute, Houston, 1955).

14. R. M. McLeod, On the zeros of the derivatives of some entire functions, Trans. Amer. Math. Soc. 91 (1959), 354-367.

15. G. Pólya, Über die Nullstellen sukzessiver Derivierten, Math. Z. 12 (1922), 36-60.

16. G. POLYA, On the zeros of the derivatives of a function and its analytic character, Bull. Amer. Math. Soc. 49 (1943), 178-191.

17. S. Saks and A. Zygmund, Analytic Functions (Elsevier Pub. Co., New York, 1971).

18. E. C. Titchmarsh, The Theory of Functions (Oxford University Press, 1939).

NORTHERN ILLINOIS UNIVERSITY

DeKaLb, IllinOIS 60115

USA 